\title{
Detecting independence in viability selection on two traits
}

\author{
HANS-ROLF GREGORIUS* \& MARTIN ZIEHE \\ Abteilung für Forstgenetik und Forstpflanzenzüchtung, Universität Göttingen, Büsgenweg 2, D-37077 Göttingen, \\ Germany
}

\begin{abstract}
Any study of interdependence of viability selection on multiple traits (or gene loci) decisively rests on the applied measure of viability selection. An appropriate measure is obtained by normalizing the survival probabilities by their maximum. A necessary and sufficient characterization of independent operation of viability selection on two traits is given. The characterization comprises both simultaneous and successive selection, and it takes account of the bias introduced by stochastic association between the traits. It is proven that every type of independent selection on two traits can be represented in the form of two successive and independent selection steps (sequential selection). The relations between independent viability selection and the resulting population reduction (the selection load) are established. An analysis of published data on viability selection in beech revealed unexpectedly large amounts of selective interaction between two enzyme loci. The considerable accompanying increase in genetic load adds an interesting aspect to the selectionist-neutralist debate.
\end{abstract}

Keywords: beech, enzymes, interaction, multiple loci, viability selection.

\section{Introduction}

Studies of joint adaptation of multiple traits play a basic role in ecological and evolutionary genetics (Stern \& Roche, 1974, chapters II and IV). Referring to the selective forces underlying the adaptative processes, the detection of interaction or independence of the selective forces acting on two traits is thus of central concern in such studies. Coadaptation, for example, constitutes an extreme case of dependent selection (coselection, selective interaction) in that the favouring of a particular association of trait states eventually leads to fixation of both of these states. Other forms of coselection may produce stable polymorphisms for multiple traits (Karlin, 1975, 1978; Nagylaki, 1992, p. 185ff; Gregorius, 1992). The same results may of course be obtained with independently selected traits; however, the larger levels of multiple trait variation that can be maintained with the additional help of recombination between the traits, may substantially contribute to adaptive flexibility. Yet, the adaptive flexibility gained by independent selection at many gene loci must be paid for by a genetic load possibly too large to guarantee population persistence. This situation is well known to have given rise to the selectionist-neutralist debate (Kimura, 1983).

*Correspondence.
The probably most common methods to reveal coselection in natural populations involve the estimation of gametic disequilibrium or correlations between traits (Weir, 1990, p. 89ff). These methods are intended to characterize the consequences of coselection without aiming explicitly at the separation of effects of direct coselection from those of other forces generating stochastic associations between traits. Such other forces may originate from special mating systems involving, for example, self-fertilization (or more generally defined genealogical mating preferences), which are known to produce persistent stochastic associations between genotypes at different gene loci without the participation of selection or drift (Bennet \& Binet, 1956). In fact, this phenomenon has again attracted much interest recently as an agent introducing a new quality into studies on the coevolution and coadaptation of traits (Strobeck, 1979).

Provided with this knowledge, it becomes more important in experimental studies of natural selection to be able to distinguish between effects that trigger selection on an observed trait (gene locus) solely by stochastic association with a directly selected background trait (sometimes called 'hitchhiking') and effects that operate directly either in combination with other traits (coselection) or independently. In order to enable this distinction, it is indispensible to build upon an unambiguous concept of independence between the 
selective forces operating on two traits. Recall that selection operating on a single trait controlled by two gene loci, say, gives rise to selection taking place simultaneously at each of these two loci, which makes it difficult to see how these two loci can be selected independently at all. This is of course different in the case of two traits expressed in temporally separated ontogenetic stages (such as juvenile and adult traits), where viability selection in the later stage may indeed operate completely independently of the selection in the earlier stage, despite the possibility of stochastic associations between the states of the two traits.

In fact, the latter type of joint selection of two traits, which will be referred to as sequential selection and is characterized by a multiplicative structure of the viabilities, appears so far to be the only explicitly considered form of selective independence (see Crow, 1970; Lewontin, 1974 , p. 201 ; occasionally both multiplicative and additive structures are viewed as forms of independent selection, see Karlin, 1978, p. 549). Yet, this does not provide us with a sufficiently general concept of independent selection as is required in the various experimental studies of viability selection in natural populations. Such a concept should rather be based on the common idea that independence in action of two variables on a joint response is realized if the effect of the state of one variable on the response is not modified by the states of the other variable. It is the purpose of the present paper to develop this concept into a concept of independent action of viability selection on two traits that circumvents the pitfalls of stochastic association between traits and, in addition, leads to a testable condition for all forms of independent selection.

The results of this basic analysis will be applied:(i) to relate sequential selection to other forms of independent selection including simultaneous selection; (ii) to derive for arbitrary selection regimes their corresponding regimes of independent selection in order to allow for the evaluation of deviations from independent selection; (iii) to quantify the effect of independent selection on population reduction (selection load); (iv) to characterize 'passive selection' as the form of selection mediated solely by stochastic associations with other directly selected traits; (v) to specify the effect of passive selection on the selection load; and (vi) to discuss data obtained for changes in isoenzyme frequencies between early stages of beech populations.

\section{Characteristics of viability selection acting independently on two traits}

Viability selection describes the situation of differential survival of the members of a collection to a specified stage. The differences in survival are determined with respect to a particular classification of the members usually described by the states of a given trait. They are then measured in terms of the proportion of surviving individuals of a given type. Hence, in addition to its type, each individual can be characterized by the state of having survived or not, and with this characterization one arrives at the notation compiled in Table 1. We decided in favour of this detailed notation to enable precise statements and proofs and to prevent the mistakes that may easily result from a more intuitive approach.

In the following $P^{*}$ will be referred to as the stochastically independent structure corresponding to $P$. It will turn out that this distribution plays a central role in the formulation of the conditions for independent selection as it eliminates effects of stochastic dependence between the traits $A$ and $B$ on the marginal viabilities for each of these traits (in fact, $P^{*}=P$ in the case of stochastic independence between $A$ and $B$ ).

Noting that the distribution of the trait $A$ before selection is given by $P(A=a)$ and after selection by $P(A=a \mid S=1)$, it follows directly from application of the standard rules of conditional probabilities that the transition equation for selection can be written in the general form:

$P(A=a \mid S=1)=P(A=a) \cdot \frac{P(S=1 \mid A=a)}{P(S=1)}$,

Table 1 Notation

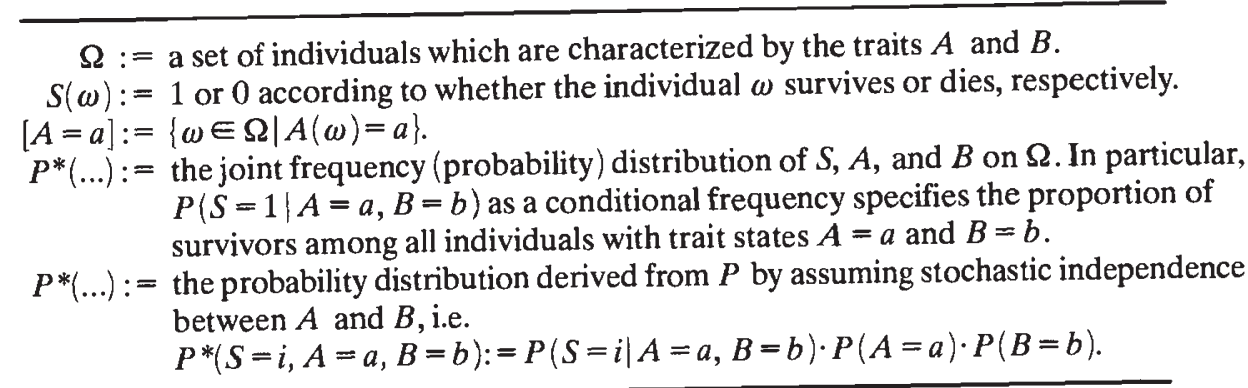


where $P(S=1 \mid A=a)$ is the fraction of surviving $A=a$-individuals, and $P(S=1)$ is the overall (average) fraction of survivors. The quotient in this equation is frequently referred to as the selective value of $A=a$ and will be denoted by:

$v_{A}(a):=\frac{P(S=1 \mid A=a)}{P(S=1)}$.

$v_{B}(b)$ is defined in the same way, as is the joint selective value:

$v(a, b):=\frac{P(S=1 \mid A=a, B=b)}{P(S=1)}$

of $A=a$ and $B=b$. This value strongly depends on stochastic associations between the two traits, as does either of the two single trait selective values. To remove this dependency, the selective values must be referred to the above-defined stochastically independent structure $P^{*}$ corresponding to $P$. The selection parameters thus obtained are again indicated by an asterisk: $v_{A}^{*}(a)$, $v_{B}^{*}(b)$, and $v^{*}(a, b)$. Note that $v(a, b)$ is identical to $v^{*}(a, b)$ only if $P(S=1)$ equals $P^{*}(S=1)$, and the latter generally fails unless structures are stochastically independent.

Yet, the selective value is of limited significance in the assessment of the intensity of selection because it is intrinsically frequency dependent via $P(S=1)$, and since the fraction of survivors (survival probability) itself includes the possibility of nonselective deaths (which are deaths in addition to those minimally required for the realized selection (Gregorius \& Degen, 1994)). For these reasons, the net selection effect is preferably measured relative to a standard set by the survival probability of one of the types involved and this type is usually one of maximum survival. The thus normalized survival probability $c_{A}(a)$,

$c_{A}(a):=\frac{P(S=1 \mid A=a)}{\max _{x} P(S=1 \mid A=x)}$

is termed the selection coefficient of $A=a . c_{B}(b)$ is defined analogously, and again $c_{A}^{*}(a)$ or $c_{B}^{*}(b)$ denote these measures in the case of corresponding stochastic independence between the traits $\left(P\right.$ replaced by $\left.P^{*}\right)$. By eliminating stochastic dependence between the traits, $c_{A}^{*}(a)$ is freed of all merely structure-induced (nonfunctional) effects possibly exerted by the $B$ trait on the $A$ trait. As pointed out by Gregorius \& Degen (1994), the standardization of viability selection parameters with respect to maximum survival has the important advantage that it again results in a probability measure defined for selective deaths only.
For a pair of jointly selected traits, $c(a, b)$ denotes the joint selection coefficient of $A=a$ and $B=b$ :

$c(a, b):=\frac{P(S=1 \mid A=a, B=b)}{\max _{x, y} P(S=1 \mid A=x, B=y)}$.

Note that in contrast to selective values, an analogously defined $c^{*}(a, b)$ would necessarily be identical to $c(a, b)$. Hence the amount of stochastic dependence has no influence on the joint selection coefficient unless the corresponding $P^{*}$ is also changed. Considering selection on the trait $A$ as possibly subject to modification by trait $B$, the conditional selection coefficient $c(a \mid b)$ of trait state $A=a$ given trait state $B=b$ is consistently defined by

$c(a \mid b):=\frac{P(S=1 \mid A=a, B=b)}{\max _{x} P(S=1 \mid A=x, B=b)}$,

and it assesses the dependency of selection at trait $A$ on trait $B$ under explicit exclusion of stochastic associations between the two traits. Proceeding from the general concept of independent action mentioned in the Introduction, one arrives at the following central definition.

The trait state $A=a$ is said to be selected independently of the trait $B$, if the conditional selection coefficient $c(a \mid b)$ of $A=a$ given $B=b$ is the same for all $b$. If this holds for all states of $A$, trait $A$ is said to be selected independently of trait $B$.

As is proven in Appendix I, this definition implies the basic characteristics of selective independence summarized in Table 2. Equation $(i)$ in this table solely refers to the stochastically independent distribution $P^{*}$ of the two traits corresponding to the initial distribution $P$. The assertion specifies that independent selection of the two traits is equivalent to the observation of stochastically independent structures of both traits among those survivors which would have remained in a population with corresponding independent structures before selection. In other words, under independent selection regimes, a structure of $P^{*}$ initially proving independence preserves the property of stochastic independence beyond the process of selection.

Equation $(i)$ can be used in statistical tests of association as it implies a comparison of the joint distribution of two variables with their corresponding stochastically independent distribution. Equations ( $i i)$ and (iii) will serve in the evaluation of differences between the estimates of the respective selection parameters (recall, however, the previous statements on the advantages of $c$ over $v$ ). Moreover, the right side of equation $(i i)$, i.e. $c_{A}^{*}(a) \cdot c_{B}^{*}(b)$, itself represents a selec- 
Table 2 Characteristics of selective independence

- Selective independence of trait $A$ from trait $B$ always implies selective independence of $B$ from $A$

- Selective independence of trait $A$ from trait $B$ is equivalent to the fulfilment of any of the following multiplicative relations holding for all $a$ and $b$ :

$$
\begin{aligned}
& P^{*}(A=a, B=b \mid S=1)=P^{*}(A=a \mid S=1) \cdot P^{*}(B=b \mid S=1) \\
& c(a, b)=c_{A}^{*}(a) \cdot c_{B}^{*}(b) \\
& v^{*}(a, b)=v_{A}^{*}(a) \cdot v_{B}^{*}(b)
\end{aligned}
$$

tion coefficient that reflects the situation of independent selection corresponding to the actual selection coefficients $c(a, b)$. The product:

$c_{\mathrm{A}}^{*}(a) \cdot c_{\mathrm{B}}^{*}(b):=c^{\circ}(a, b)$

will therefore be referred to as the coefficient of corresponding independent selection.

It is important to realize that the present characterization of independence of selection between traits vitally depends on the measurement of selection in terms of the selection coefficient as defined above. If, for example, the non-normalized survival probabilities had been used in place of the selection coefficients, a basically different formal characterization of independent selection would have resulted even when applying the same general concept of independent action. In the case of the selective value $v$, it has to be considered that the characterization of independence includes effects which are not directly related to selection, as was pointed out earlier.

\section{Sequential viability selection}

The above finding, that independent selection allows for a multiplicative representation of the viabilities with both factors determined separately by each of the two traits, resembles the situation of sequential selection mentioned in the Introduction. In other words, this finding raises the question as to whether each form of simultaneous, independent selection can be represented as two successive steps of selection, where one trait is selected at a time with no regard to the other trait. This is the idea of sequential selection and it requires a formal definition within the above framework to make explicit the kind of relation to independent selection.

Definition: The traits $A$ and $B$ are said to be sequentially selected in that order, if there exist survival variables $S_{A}, S_{B}: \Omega \rightarrow\{0,1\}$ for each trait separately and characterized by $\left[S_{A}=\emptyset, S_{B}=1\right]=\emptyset$ (failure to survive stage $A$ excludes survival of stage $B$ ) and $[S=1]=\left[S_{A}=1, S_{B}=1\right]$ (survival to the census stage requires survival of both stages $A$ and $B)$, so that

$$
\begin{aligned}
& P(S=1 \mid A=a, B=b)=P\left(S_{A}=1 \mid A=a, B=b\right) \\
& \quad P\left(S_{B}=1 \mid S_{A}=1, A=a, B=b\right),
\end{aligned}
$$

and where the last two conditional distributions fulfil the independence conditions

(i) $P\left(S_{A}=1 \mid A=a, B=b\right)=P\left(S_{A}=1 \mid A=a\right)$ and

(ii) $P\left(S_{B}=1 \mid S_{A}=1, A=a, B=b\right)$

$$
=P\left(S_{B}=1 \mid S_{A}=1, B=b\right) \text {. }
$$

Independence condition (i) simply confirms the fact that the first step of selection (of the $A$ trait) does not depend on the second step ( $B$ selection). Condition (ii) as the actual condition of independence requires that once the $A$ stage is survived, survival of the $B$ stage does not depend on the state of the $A$ trait. This representation precisely reflects the common idea that joint viability be equal to the product of the two viabilities. Comparison with the results on independent selection summarized in Table 2 shows (as proven in Appendix II) that both concepts (independent and sequential selection) indeed describe the same phenomenon. More precisely:

Two traits $A$ and $B$ are independently selected if and only if they are sequentially selected. In the case of independent selection, survival probabilities of a corresponding system of sequential selection (designated by the joint frequency distribution $P^{\prime}$ ) are specified by equations (i) to (iv) of Appendix II.

Recall that the sequential representation of independent selection includes cases in which selection primarily acts on a single trait which, in turn, is under complete control of another pair of traits (gene loci, for example). Hence, selection taking place simultaneously and independently on two traits can always be conceived of as occurring in two successive and independent steps. Which of the two traits is considered to be selected first is irrelevant. All survival frequencies in the corresponding sequential representation are 
uniquely determined once the overall fraction of survivors of the first step of selection $\left(P^{\prime}\left(S_{A}=1\right)\right.$ if trait $A$ marks this first step) is chosen within the interval specified in condition (iv) in Appendix II. The possibility to choose either of the two traits to represent the first selection episode together with the arbitrary specifiability of the overall fraction of survivors of this episode within certain limits emphasizes, however, that the correspondence between the two forms of independent selection is not complete: a particular situation of simultaneous, independent selection may summarize a host of corresponding sequential selection regimes.

\section{Selection load and independent selection}

Gregorius \& Degen (1994) noted that for a specified trait the viability selection load measures the minimal reduction in population size required for the change in state frequencies brought about by the selection. As surplus deaths have no bearing on the state frequencies, the (viability) selection load may be conceived of as accounting only for selective deaths. Viability selection load as determined by adaptive requirements is thus a basic parameter of population persistence. What are the effects of co- or independent selection on this parameter?

For trait $A$, say, the selection load $L_{A}$ is given by

$L_{A}:=1-\frac{P(S=1)}{\max _{a} P(S=1 \mid A=a)}=1-\sum_{a} c_{A}(a) \cdot P(A=a)$.

$L_{B}$ and $L_{A B}$ are defined accordingly. With reference to the distribution $P^{*}$ corresponding to stochastically independent association between $A$ and $B$, the selection load $L_{A}^{*}$ takes the form:

$L_{A}^{*}:=1-\frac{P^{*}(S=1)}{\max _{a} P^{*}(S=1 \mid A=a)}=1-\sum_{a} c_{A}^{*}(a) \cdot P(A=a)$.
$L_{B}^{*}$ as well as $L_{A B}^{*}$ are defined analogously. A difference, however, must be made between $L_{A B}^{*}$ and $L_{A B}^{\circ}$, the latter specifying the selection load for corresponding independent selection by:

$L_{A B}^{\circ}:=1-\sum_{a, b} c^{\circ}(a, b) \cdot P(A=a, B=b)$.

A number of basic characteristics of the selection load relevant to the present topic are summarized in Table 3. In this table assertion (i) follows directly from the fact that:

$$
\begin{aligned}
& \max _{a, b} P(S=1 \mid A=a, B=b) \\
& \quad \geq \max \left\{\max _{a} P(S=1 \mid A=a), \max _{b} P(S=1 \mid B=b)\right\},
\end{aligned}
$$

while (ii) is a consequence of eqn (2) in Appendix I.

The results in Table 3 confirm that the multiplicative structure imposed by selective independence on the viabilities carries over to the overall selective survival (the complement of the load). Stochastic dependence between the two traits increases or lowers the overall selective survival according to whether this dependence leads to $P(S=1)>P^{*}(S=1)$ or $P(S=1)<P^{*}(S=1)$ (second equation in assertion (ii)). This finding, and in particular that for both selective and stochastic independence between the two traits (assertion (iii)), underlies the selectionist-neutralist debate because, when iterated over multiple traits, it predicts drastic population reductions for large numbers of independently selected traits (gene loci). Hence, if selection were nevertheless observed on many traits (and there are many reports supporting this assumption), persistence of a population can be warranted only if either most of these traits are coselected or are 'passively' selected. The characterization and the effects of the latter are considered in the next section.

Table 3 Characteristics of the selection load

(i) Multiple trait selection loads and their component single trait loads are related by the basic inequality:

$L_{A B} \geq \max \left\{L_{A}, L_{B}\right\}$.

(ii) Selective independence between $A$ and $B$ implies the two equivalent relations

$$
\begin{aligned}
& 1-L_{A B}^{*}=1-L_{A B}^{\circ}=\left(1-L_{A}^{*}\right) \cdot\left(1-L_{B}^{*}\right) \text { and } \\
& 1-L_{A B}=\left(1-L_{A}^{*}\right) \cdot\left(1-L_{B}^{*}\right) \cdot \frac{P(S=1)}{P^{*}(S=1)} .
\end{aligned}
$$

(iii) Selective independence combined with stochastic independence between $A$ and $B$ implies

$$
1-L_{A B}=\left(1-L_{A}\right) \cdot\left(1-L_{B}\right) \text {. }
$$




\section{Passive selection}

As was emphasized in the Introduction, selection on any one trait may merely be the result of stochastic association with other traits on which selection operates directly. To characterize this type of selection, it is again necessary to consider joint selection on at least two traits, one of which is assumed to be directly selected and the other to show no selection that cannot be attributed to the first trait. This leads us to the following definition.

Definition: The trait $A$ is said to be conditionally selectively neutral with respect to the trait state $B=b$, if no conditional selection acts on $A$ for given $B=b$, i.e. if $P(S=1 \mid A=a, B=b)=P(S=1 \mid B=b)$ for all a. If, in addition, this equality holds for all $b$, conditional selective neutrality of $A$ with respect to $B$ is realized. In case $A$ and $B$ are selected but $A$ is conditionally selectively neutral with respect to $B$, then $A$ is said to be passively selected by $B$.

This concept of conditional selective neutrality allows for a number of conclusions which are of considerable value for studies of the extent of passive viability selection in natural populations. These conclusions are summarized in Table 4 and are proven in Appendix III.

An important conclusion to be drawn from assertion (i) in Table 4 is that the observation of selection on two traits excludes the possibility of mutual passive selection between these traits. Assertion (ii) confirms the expectation that passive selection does not contribute to population survival in terms of the selection load, and (iii) makes this statement more concrete in that it identifies selection loads observed at passively selected traits as minimum estimates of the overall selective population reduction (see also Gregorius \& Degen, 1994). Assertion (iv), finally, qualifies passive selection as a special form of independent selection which elimi- nates the effects of stochastic association between the traits on the directly selected trait $(B)$ and on the overall survival, thereby generating no additional selection load. This accords with assertion (ii) and the expectation advanced at the end of the last section.

\section{Discussion}

The concept of independent selection regimes underlies more or less explicitly all efforts to support the suggestion that the amount of observed genetic variation is too large to be explained essentially by balancing selection. Overdominance, when operating independently at a large number of polymorphic gene loci, would lead to an extreme genetic load and thus to an extreme reduction in population size (see e.g. Crow, 1970 , p. 154 , or Lewontin, 1974 , p. 201). An additional assumption implicit in these considerations is stochastic independence between loci. Even though largely ignored, this assumption is essential to the load argument, as granted selective independence, stochastic associations can substantially reduce the genetic load. As is well known, mixed-mating systems, for example, can generate persistent stochastic associations over the gene loci (Bennet \& Binet, 1956) which may imply considerable amounts of passive selection (Brown, 1979; Strobeck, 1979; Charlesworth, 1991; Ziehe \& Müller-Starck, 1992; Ziehe, unpublished data).

Yet, little seems to be known about whether selective interaction and stochastic associations among loci as well as their joint action play a significant role in shaping the genetic structures and loads in real populations. To demonstrate the application of the abovedeveloped methods of multilocus analysis and to see whether they provide means for revealing aspects of selection probably not recognizable so far with common single locus or multiple locus methods of analysis (the latter chiefly referring to the estimation of gametic

Table 4 Characteristics of conditional selective neutrality

(i) If $A$ and $B$ are conditionally selectively neutral with respect to each other, then selection is absent from both traits.

(ii) Passive selection has no effect on the overall viability in the sense that the selective part of the population reduction (i.e. the selection load) is completely determined by the non-passively selected traits $\left(L_{A B}=L_{B}\right.$ for $A$ conditionally selectively neutral with respect to $B$ ).

(iii) The selection loads on passively selected traits can never exceed the selection loads on the traits they depend on $\left(L_{A} \leq L_{B}\right.$ for $A$ passively selected by $\left.B\right)$.

(iv) Passive selection of $A$ by $B$ is a special form of independent selection between these two traits, and it is characterized by:

- $P^{*}(S=1 \mid A=a)=P(S=1)=P^{*}(S=1)$ for all $a$, - $P^{*}(S=1 \mid B=b)=P(S=1 \mid B=b)$ for all $b$,

- $L_{A}^{*}=0$, and $L_{A B}^{*}=L_{A B}=L_{A B}^{\circ}=L_{B}=L_{B}^{*}$. 
disequilibria), we decided to employ these methods for the interpretation of published results on viability selection in early stages of beech.

\section{Selection at two isozyme gene loci in seedlings of beech (Fagus sylvatica L.)}

The data on genotype frequencies at two isozyme loci ( $I D H-A$ and $M D H-C$, referred to as $A$ and $B$ loci in the following) in Table 5 are taken from a study by Müller-Starck (1993) on viability selection in beech seedlings (Fagus sylvatica L.). The study is based on two random samples of beech nuts from a German provenance (Nidda). The first sample of 173 seeds served for estimating genotypic frequencies at a number of enzyme loci in the second sample of 1500 beech nuts, which was exposed to field conditions (at a site close to the city of Arnsberg, Germany). After 2 years under these conditions, genotypic frequencies among the 122 surviving seedlings were determined for the same enzyme loci. The results provide estimations of genotypic structures before and after an episode of early viability selection. The genotypic frequencies in Table 5 are complemented by the stochastically independent structure corresponding to the seed frequencies and by the pertaining single and multilocus selection coefficients.

Among the observations most relevant to the present topic on the effects of selective and stochastic associations between traits are the following.

1 Single locus selection. Ignoring the dependency of selection at one locus on other loci (i.e. single locus analysis), the present data provide maximum survival probability for $A_{2} A_{2}$ at the $A$ locus and $B_{1} B_{2}$ at the $B$ locus. However, selection as measured by the selection potential $\max _{a}\left\{1-c_{A}(a)\right\}$ at the $A$ locus proves to be quite small $(0.053$, i.e. 5.3 per cent $)$. The larger value for the selection potential $\max _{b}\left\{1-c_{B}(b)\right\}$ at the $B$ locus $(0.204)$ is determined by the rare genotype $B_{1} B_{1}$ and is therefore more prone to sampling error. (Note that the selection potential is the upper limit of the selection load - and thus selective reduction - attainable for a given regime of selection coefficients.)

2 Stochastic associations. To assess the stochastic associations, the seed structure has to be compared with the corresponding expectations under stochastic independence of the single locus structures. For the present example, pronounced deviations from stochastic independence between loci can be found in the seed sample. These deviations are concentrated on the

Table 5 Frequencies and selection coefficients among beech nuts and seedlings at two enzyme gene loci

\begin{tabular}{|c|c|c|c|c|c|c|}
\hline \multirow{2}{*}{$\begin{array}{l}\text { Locus } B \\
(M D H-C)\end{array}$} & & \multicolumn{3}{|c|}{ Locus $A(I D H-A)$} & \multirow[b]{2}{*}{$c_{B}$} & \multirow[b]{2}{*}{$c_{B}^{*}$} \\
\hline & & $A_{2} A_{2}$ & $A_{2} A_{3}$ & $A_{3} A_{3}$ & & \\
\hline$B_{1} B_{1}$ & $\begin{array}{l}\text { Seed } \\
\text { Independent structure } \\
\text { Seedlings } \\
c \\
c^{\circ}\end{array}$ & $\begin{array}{l}2 \\
1.99 \\
1 \\
43.8 \\
72.9\end{array}$ & $\begin{array}{l}7 \\
6.07 \\
3 \\
37.5 \\
76.8\end{array}$ & $\begin{array}{l}6 \\
6.94 \\
5 \\
72.9 \\
71.3\end{array}$ & 79.6 & 76.8 \\
\hline$B_{1} B_{2}$ & $\begin{array}{l}\text { Seed } \\
\text { Independent structure } \\
\text { Seedlings } \\
c \\
c^{\circ}\end{array}$ & $\begin{array}{l}13 \\
9.71 \\
10 \\
67.3 \\
94.8\end{array}$ & $\begin{array}{l}21 \\
29.54 \\
24 \\
100 \\
100\end{array}$ & $\begin{array}{l}39 \\
33.76 \\
21 \\
47.1 \\
92.8\end{array}$ & 100.01 & 100.0 \\
\hline$B_{2} B_{2}$ & $\begin{array}{l}\text { Seed } \\
\text { Independent structure } \\
\text { Seedlings } \\
c \\
c^{\circ}\end{array}$ & $\begin{array}{c}8 \\
11.3 \\
6 \\
65.6 \\
82.5\end{array}$ & $\begin{array}{l}42 \\
34.39 \\
22 \\
45.8 \\
87.0\end{array}$ & $\begin{array}{l}35 \\
39.31 \\
30 \\
75.0 \\
80.7\end{array}$ & 90.6 & 87.0 \\
\hline & $\begin{array}{l}c_{A} \\
c_{A}^{*}\end{array}$ & $\begin{array}{c}100 \\
94.8\end{array}$ & $\begin{array}{c}94.7 \\
100\end{array}$ & $\begin{array}{l}94.7 \\
92.8\end{array}$ & & \\
\hline
\end{tabular}

Selection coefficients are specified as percentages; $c:=$ two locus selection coefficient; $c^{\circ}:=$ two locus coefficient of corresponding independent selection; $c_{A}$, $c_{B}:=$ single locus selection coefficients; $c_{A}^{*}, c_{B}^{*}:=$ single locus selection coefficients for corresponding stochastic independence. 
frequent two locus genotypes $A_{2} A_{3} B_{1} B_{2}, A_{2} A_{3} B_{2} B_{2}$, $A_{3} A_{3} B_{1} B_{2}$ and $A_{3} A_{3} B_{2} B_{2}$. Passive effects can therefore be expected to play a considerable role.

3 Stochastic associations and single locus selection. As shown above, elimination of the effects of passive selection exerted by the observed stochastic associations leads to the selection coefficients $c_{A}^{*}$ and $c_{B}^{*}$. These have to be compared with $c_{A}$ and $c_{B}$ to analyse the effects of passive selection. At gene locus $A$, passive selection apparently shifted the maximum viability from the heterozygote $A_{2} A_{3}$ to the homozygote $A_{2} A_{2}$ and lowered the selection potential slightly from $\max _{a}\left\{1-c_{A}^{*}(a)\right\}=0.072$ to 0.053 . For the $B$ locus, ranking of genotype viabilities is not modified by stochastic association but selection potential is again slightly lowered from $\max _{b}\left\{1-c_{B}^{*}(b)\right\}=0.232$ to 0.204 .

4 Selective associations. To discuss deviations from independent selection, the two locus selection coefficients $c$ have to be compared with the $c^{\circ}$ s (specifying the coefficients of corresponding independent selection). Apparently, as a result of selective interaction, genotype $A_{3} A_{3}$ suffers a strong disadvantage when associated with $B_{1} B_{2}$, despite the fact that the heterozygote $B_{1} B_{2}$ is the most viable $B$ locus genotype; the selection coefficient amounts to almost only half of the coefficient of corresponding independent selection in this case. Similarly, $A_{2} A_{3}$ combined with $B_{2} B_{2}$ demonstrates strong deviation from corresponding independent selection. Hence, pronounced selective interaction between the two loci can be stated. The interaction is remarkably consistent with respect to the ranking between the selection coefficients $c$ and $c^{\circ}$ (the only exception occurring for a rare genotype) with the result of intensifying selection. This is confirmed by the fact that $L_{A B}=0.383>L_{A B}^{\circ}=0.121$.

These observations reveal a striking discrepancy between single and two locus selection in that the two locus selection potential exceeds the single locus potentials by orders of magnitude. Possibly because larger single locus selection potentials would have been required, the notable stochastic associations did not translate into noticeable degrees of passive selection. This is corroborated by the selection loads, which show single locus values of $L_{A}=0.046$ and $L_{B}=0.064$ compared with a two locus value of $L_{A B}=0.383$.

Moreover, the above-discussed possibility that selective interaction and stochastic dependence between loci (as found in the present data) might reduce the genetic load below that expected from independent action cannot be confirmed. $L_{A}^{*}=0.04$ and $L_{B}^{*}=0.084$ indicate relatively slight single locus effects if the influence of stochastic associations is eliminated, and in fact $L_{A B}^{*}$ as well as $L_{A B}^{\circ}$ are both smaller than $L_{A B}$. Whereas the two locus load is slightly increased by stochastic association $\left(L_{A B}=0.383>L_{A B}^{*}=0.348\right)$, selective interaction drastically increases the load (from $L_{A B}^{\circ}=0.121$ to $L_{A B}=0.383$ ). Without further analysis this finding seems to augment rather than solve the dilemma of excessive selection required for the maintenance of the observed amounts of genetic variation.

The profound degrees of selective interaction observed between the two loci might give rise to the speculation that forces other than selection operating directly on these loci are unlikely causes. The fact that the enzyme systems IDH and MDH are both involved in the citric acid cycle could be quoted as evidence supporting this hypothesis. On the other hand, in principle, it cannot be excluded that passive selection by the operative genetic background may have effected strong (passive) selective interaction between our loci. This could be brought about by stochastic association of each of the two loci with distinct operative and selectively interacting parts of the genetic background. If this were true, it would also hint at the possibility of coselection in sizable parts of the genome.

Another possible explanation of the present observations can be derived from the fact that the material studied does not descend from any of the local populations. This implies the possibility of novel selection pressures. As the presence of major polymorphisms at both loci suggests stabilizing selection in the original populations, the question now is whether these selective forces continue in the new environment. The single locus selection coefficients do not contradict such continuation because they represent overdominance. Yet, the ranking of the observed two locus selection coefficients invalidates the conditions for protected two locus polymorphisms (Gregorius, 1991; condition (5)). Hence the novel environmental conditions appear to destabilize the established polymorphism via selective interaction between the two loci.

\section{Acknowledgements}

This work was financially supported by grant $\mathrm{Gr} 435$ / 12-1 from the Deutsche Forschungsgemeinschaft, Bonn, Germany.

\section{References}

BENNET, J. H. AND BINET, F. E. 1956. Association between Mendelian factors with mixed selfing and random mating. Heredity, 10, 51-56.

BRown, A. H. D. 1979. Enzyme polymorphism in plant populations. Theor. Pop. Biol., 15, 1-42. 
CHARLESWORTH, D. 1991. The apparent selection on neutral marker loci in partially inbreeding populations. Genet. Res., 57, 159-175.

CROW, J. F. 1970. Genetic loads and the cost of natural selection. In: Kojima, K. (ed.) Mathematical Topics in Population Genetics. pp. 128-177. Spinger, Berlin, Heidelberg.

GREGORIUS, H.-R. 1991. Establishment of allelic two-locus polymorphisms. J. Math. Biol., 30, 185-197.

GREGORIUS, H.-R. AND DEGEN, B. 1994. Estimating the extent of natural selection in seedlings from different Fagus sylvatica (L.) populations: application of new measures. J. Hered., 85, 183-190.

KARLIN, s. 1975. General two-locus selection models: some objectives, results and interpretations. Theor. Pop. Biol., 7, 364-398.

KARLIN, S. 1978. Theoretical aspects of multi-locus selection balance. I. In: Levin, S. A. (ed.) Studies in Mathematics, vol. 16. Studies in Mathematical Biology, pp. 503-587. The Mathematical Association of America, Washington.

KIMURA, M. 1983. The Neutral Theory of Molecular Evolution. Cambridge University Press, Cambridge.

LEWontin, R. C. 1974. The Genetic Basis of Evolutionary Change. Columbia University Press, New York

MULlER-STARCK, G. 1993. Auswirkungen von Umweltbelastungen auf genetische Strukturen von Waldbeständen am Beispiel der Buche (Fagus sylvatica L.) Schriften aus der Forstl. Fak. d. Universität Göttingen u. d. Niedersächs. Forstl. Versuchsanst., Bd. 112. J. D. Sauerländer's Verlag, Frankfurt am Main (in German with English Summary).

NAGYLAKl, T. 1992. Introduction to Theoretical Population Genetics. Springer, Berlin, Heidelberg.

STERN, K. AND ROCHE, L. 1974. Genetics of Forest Ecosystems. Springer, Berlin, Heidelberg.

STROBECK, C. 1979. Partial selfing and linkage: the effect of a heterotic locus on a neutral locus. Genetics, 92, 305-315.

WEIR, B. S. 1990. Genetic Data Analysis. Sinauer Associates, Sunderland, MA.

ZIEHE, M. AND MUller-STARCK, G. 1992. Changes of genetic variation due to associated selection. In: Müller-Starck, G. and Ziehe, M. (eds) Genetic Variation in European Populations of Forest Trees, pp. 259-271. J. D. Sauerländer's Verlag, Frankfurt am Main.

\section{Appendix I}

Firstly, it will be shown that selective independence of trait state $A=a$ from trait $B$ is equivalent to:

$$
\begin{aligned}
P(S=1 \mid A=a, B=b) \\
\quad=\frac{P^{*}(S=1 \mid A=a) \cdot \max _{x} P(S=1 \mid A=x, B=b)}{\Sigma_{y} P(B=y) \cdot \max _{x} P(S=1 \mid A=x, B=y)}
\end{aligned}
$$

for all $b$. By definition of selective independence of $A=a$ from $B$ there exists a constant $c_{a}$ such that $P(S=1 \mid A=a, B=b)=c_{a} \cdot \max _{x} P(S=1 \mid A=x, B=b)$ for all $b$. Multiplication of both sides of this equality with $P(B=b)$ and summation over $b$ yields $P^{*}(S=1 \mid A=a)=c_{a} \cdot \Sigma_{b} P(B=b) \cdot \max _{x} P(S=1 \mid A=x$, $B=b)$, from which eqn (1) immediately follows. Applying eqn (1) it is easily seen that $c(a \mid b)$ does not depend on $b$, which proves the reverse direction.

Next, it will be shown that selective independence of trait $A$ from trait $B$ is equivalent to:

$$
\begin{aligned}
& P(S=1 \mid A=a, B=b) \cdot P^{*}(S=1) \\
& \quad=P^{*}(S=1 \mid A=a) \cdot P^{*}(S=1 \mid B=b)
\end{aligned}
$$

for all $a$ and $b$. If $A$ is selected independently from $B$, eqn (1) holds for all $a$ and one obtains

$\frac{P\left(S=1 \mid A=a_{1}, B=b\right)}{P\left(S=1 \mid A=a_{2}, B=b\right)}=\frac{P^{*}\left(S=1 \mid A=a_{1}\right)}{P^{*}\left(S=1 \mid A=a_{2}\right)}$.

Thus $\quad P\left(S=1 \mid A=a_{1}, \quad B=b\right) \cdot P^{*}\left(S=1 \mid A=a_{2}\right)$ $=P\left(S=1 \mid A=a_{2}, B=b\right) \cdot P^{*}\left(S=1 \mid A=a_{1}\right)$. Multiplication of both sides with $P\left(A=a_{2}\right)$ and summation over $a_{2}$ yields $P(S=1 \mid A=a, B=b)$ - $P^{*}(S=1)=P^{*}(S=1 \mid B=\mathrm{b}) \cdot P^{*}(S=1 \mid A=a) \quad$ and thus eqn (2). The reverse direction follows from $\max _{a} P(S=1 \mid A=a, B=b)$

$=\frac{P^{*}(S=1 \mid B=b)}{P^{*}(S=1)} \cdot \max _{a} P^{*}(S=1 \mid A=a)$.

From the symmetry of the arguments in eqn (2) it can immediately be taken that independence of $A$ from $B$ is equivalent to independence of $B$ from $A$.

$\operatorname{Eqn}(2)$ is equivalently transformed into:

$$
\begin{aligned}
& P^{*}(A=a, B=b \mid S=1) \\
& \quad=P^{*}(A=a \mid S=1) \cdot P^{*}(B=b \mid S=1),
\end{aligned}
$$

which confirms the multiplicative relation (i) in Table 2 to be equivalent to independent selection.

Now assume independent selection. Eqn (2) implies:

$$
\begin{aligned}
\max _{x, y} P(S=1 \mid A=x, B=y) \cdot P^{*}(S=1) \\
\quad=\left[\max _{x} P^{*}(S=1 \mid A=x)\right] \cdot\left[\max _{y} P^{*}(S=1 \mid B=y)\right]
\end{aligned}
$$

and eqn (2) together with eqn (4) yields the decomposition (ii) in Table 2 for the joint selection coefficients. To prove the reverse direction, assume the decomposition (ii) of selection coefficients. Summing up over all trait states of $A$ and $B$ gives eqn (4). Applying this to the decomposition equation results in eqn (1), which is equivalent to independent selection.

Equation (2) is easily transformed into $v^{*}(a, b)=v_{A}^{*}(a) \cdot v_{B}^{*}(b)$, which proves that the decomposition (iii) in Table 2 of selective values is also equivalent to independent selection. 


\section{Appendix II}

Let $A$ and $B$ be sequentially selected, then:

$\frac{P(S=1 \mid A=a, B=b)}{\max _{x} P(S=1 \mid A=x, B=b)}=\frac{P\left(S_{A}=1 \mid A=a\right)}{\max _{x} P\left(S_{A}=1 \mid A=x\right)}$

which is independent of $b$ and therefore proves that $A$ is selected independently of $B$. Hence $A$ and $B$ are selected independently.

To prove the reverse direction suppose that $A$ and $B$ are selected independently and consider survival variables $S_{A}, S_{B}: \Omega \rightarrow\{0,1\}$ with $S_{A} \cdot S_{B}=S$ and joint distribution $P^{\prime}$ characterized by:

(i) $P^{\prime}(S=i, A=a, B=b)=P(S=i, A=a, B=b)$,

(ii) $P^{\prime}\left(S_{A}=1 \mid A=a, B=b\right)$

$$
=P^{\prime}\left(S_{A}=1\right) \cdot \frac{P^{*}(S=1 \mid A=a)}{P^{*}(S=1)},
$$

(iii) $P^{\prime}\left(S_{B}=1 \mid S_{A}=1, A=a, B=b\right)$

$$
=\frac{P^{*}(S=1 \mid B=b)}{P^{\prime}\left(S_{A}=1\right)} .
$$

In these expressions $P^{\prime}\left(S_{A}=1\right)$ must satisfy the inequality:

(iv) $\max _{b} P^{*}(S=1 \mid B=b) \leq P^{\prime}\left(S_{A}=1\right)$

$$
\leq \frac{P^{*}(S=1)}{\max _{a} P^{*}(S=1 \mid A=a)}
$$

to guarantee that the $P^{\prime}$ values do not exceed 1 . That the implicit inequality $\max _{b} P^{*}(S=1 \mid B=b)$

$\leq \frac{P^{*}(S=1)}{\max _{a} P^{*}(S=1 \mid A=a)}$

is indeed fulfilled follows from the assumption of selective independence, which, by making use of eqn (2) in Appendix I, implies:

$$
\begin{aligned}
1 & \geq \max _{a, b} P(S=1 \mid A=a, B=b) \\
& =\max _{a, b} \frac{P^{*}(S=1 \mid A=a) \cdot P^{*}(S=1 \mid B=b)}{P^{*}(S=1)} \\
& =\frac{\max _{a} P^{*}(S=1 \mid A=a) \cdot \max _{b} P^{*}(S=1 \mid B=b)}{P^{*}(S=1)} .
\end{aligned}
$$

Thus, any choice of $P^{\prime}\left(S_{A}=1\right)$ within the above bounds specifies a well-defined distribution $P^{\prime}$ of sequential selection that coincides with the initial distribution $P$ of the variables $S, A$ and $B$, and in which trait $A$ is selected prior to trait $B$. This completes the proof.

\section{Appendix III}

The proof of assertions (i) and (iv) in Table 4 is straightforward. Assertion (ii) follows from the fact that, for a trait $A$ which is conditionally selectively neutral with respect to a trait $B, \max _{a, b} P(S=1 \mid A=a$, $B=b)=\max _{b} P(S=1 \mid B=b)$ holds, so that $L_{A B}=L_{B}$. In addition, $L_{A B} \geq \max \left\{L_{A}, L_{B}\right\}$, as shown above, so that $L_{A} \leq L_{B}$, which proves assertion (iii). 